\title{
NEW RAMANUJAN-KOLBERG TYPE PARTITION IDENTITIES
}

\author{
Heng Huat Chan, Heekyoung Hahn, Richard P. Lewis, \\ AND SiEW Lian TAN
}

\begin{abstract}
In this article, we use functions studied by N. J. Fine and R. J. Evans to construct analogues of modular equations first discovered by S. Ramanujan. We then use these functions to construct new identities satisfied by $\sum_{n=0}^{\infty} p(\ln +k) q^{n}$, with odd prime $l$ and $0 \leq k \leq(l-1)$. Our new partition identities are inspired by the work of O. Kolberg and Ramanujan.
\end{abstract}

\section{Introduction}

Let $p(n)$ denote the number of unrestricted partitions of the non-negative integer $n$. Let $l$ and $k$ be non-negative integers and set

$$
P_{l, k}(q):=\sum_{n=0}^{\infty} p(\ln +k) q^{n}, \quad|q|<1 .
$$

In [13], S. Ramanujan stated and sketched the proofs of the identities

$$
P_{5,4}(q)=5 \prod_{n=1}^{\infty} \frac{\left(1-q^{5 n}\right)^{5}}{\left(1-q^{n}\right)^{6}}
$$

and

$$
P_{7,5}(q)=7 \prod_{n=1}^{\infty} \frac{\left(1-q^{7 n}\right)^{3}}{\left(1-q^{n}\right)^{4}}+49 q \prod_{n=1}^{\infty} \frac{\left(1-q^{7 n}\right)^{7}}{\left(1-q^{n}\right)^{8}} .
$$

Ramanujan then deduced from (1.1) and (1.2) that

$$
\begin{aligned}
& p(5 n+4) \equiv 0(\bmod 5), p(25 n+24) \equiv 0(\bmod 25), \\
& p(7 n+5) \equiv 0(\bmod 7) \text { and } p(49 n+47) \equiv 0(\bmod 49) .
\end{aligned}
$$

Received July 22, 2002.

The first author is funded by National University of Singapore Academic Research Fund, Project Number R14000027112. 
Around 1939, H.S. Zuckerman discovered the analogue of (1.1) and (1.2) for $l=13$ and $k=6$, namely,

$$
\begin{aligned}
P_{13,6}(q)= & 11 \prod_{n=1}^{\infty} \frac{\left(1-q^{13 n}\right)}{\left(1-q^{n}\right)^{2}}+468 q \prod_{n=1}^{\infty} \frac{\left(1-q^{13 n}\right)^{3}}{\left(1-q^{n}\right)^{4}}+6422 q^{2} \prod_{n=1}^{\infty} \frac{\left(1-q^{13 n}\right)^{5}}{\left(1-q^{n}\right)^{6}} \\
& +43940 q^{3} \prod_{n=1}^{\infty} \frac{\left(1-q^{13 n}\right)^{7}}{\left(1-q^{n}\right)^{8}}+171366 q^{4} \prod_{n=1}^{\infty} \frac{\left(1-q^{13 n}\right)^{9}}{\left(1-q^{n}\right)^{10}} \\
& +371293 q^{5} \prod_{n=1}^{\infty} \frac{\left(1-q^{13 n}\right)^{11}}{\left(1-q^{n}\right)^{12}}+371293 q^{6} \prod_{n=1}^{\infty} \frac{\left(1-q^{13 n}\right)^{13}}{\left(1-q^{n}\right)^{14}} .
\end{aligned}
$$

The pairs $(l, k)=(5,4),(7,5)$, and $(13,6)$ in $(1.1)-(1.3)$ satisfy the congruence

$$
24 k \equiv 1 \quad(\bmod l) .
$$

From now on, we will denote the integer $1 \leq k \leq(l-1)$ satisfying (1.4) by $\delta_{l}$.

When $k \neq \delta_{l}$, formulas for $P_{l, k}$ are much more complicated. For example, for $l=7$ and $k=0$, A. B. Ekin [5] discovered that

$$
\mathcal{P}_{7,0}(q) W_{7,3}=W_{7,3}^{7}-W_{7,3}^{5} W_{7,1}+17 W_{7,3}^{3} W_{7,1}^{2}+10 W_{7,3} W_{7,1}^{3}+2 W_{7,3}^{-1} W_{7,1}^{4},
$$

where

$$
\begin{aligned}
W_{l, j}=W_{l, j}(z)=q^{\frac{6 j^{2}}{l}-j} \prod_{n=1}^{\infty} \frac{\left(1-q^{l(n-1)+4 j}\right)\left(1-q^{l n-4 j}\right)}{\left(1-q^{l(n-1)+2 j}\right)\left(1-q^{l n-2 j}\right)} & \\
& q=e^{2 \pi i z}, 1 \leq j \leq \frac{l-1}{2},
\end{aligned}
$$

and

$$
\begin{aligned}
\mathcal{P}_{l, k}=\mathcal{P}_{l, k}(q)=q^{\left(l^{2}+l-l^{3}+24 k-1\right) /(24 l)} \prod_{n=1}^{\infty} \frac{\left(1-q^{n}\right)^{l+1}}{\left(1-q^{l n}\right)^{l}} P_{l, k}(q), & \\
& 0 \leq k \leq(l-1) .
\end{aligned}
$$

Besides (1.5), Ekin also discovered identities for $\mathcal{P}_{7, i}$ for $i=1,2,3,4$ and 6 . Ekin's identities are not surprising in view of O. Kolberg's identity (see $[9$, Lemma 4]). However, his identities are simpler than those obtained from Kolberg's formula. It was from Ekin's identities that R. P. Lewis [11] noticed that the group $\Gamma_{0}(7)$ acts on $\left\{\mathcal{P}_{7, i} \mid 0 \leq k \leq 6\right\}$ giving the orbits $\left\{\mathcal{P}_{7,0}, \mathcal{P}_{7,2}, \mathcal{P}_{7,6}\right\}$, $\left\{\mathcal{P}_{7,1}, \mathcal{P}_{7,3}, \mathcal{P}_{7,4}\right\}$ and $\left\{\mathcal{P}_{7,5}\right\}$. He proved that for every prime $l>3$, the group $\Gamma_{0}(l)$ acts on $\left\{\mathcal{P}_{l, k} \mid 0 \leq k \leq l-1\right\}$ and partitions this set into three orbits determined by the Jacobi symbol $\left(\frac{24 k-1}{6 l}\right)$. Note that there is always one orbit that contains a single element, namely, the one with $k=\delta_{l}$.

The evidence that $\left\{\mathcal{P}_{l, k}\right\}$ can be partitioned into these three sets as indicated by Lewis can be found in Kolberg's paper. For example, Kolberg proved that 
$[9$, eqs. $(5.2),(5.3)]$

$$
\mathcal{P}_{7,1} \mathcal{P}_{7,3} \mathcal{P}_{7,4}=15 h_{7}^{4}+12 \cdot 7^{2} h_{7}^{3}+24 \cdot 7^{3} h_{7}^{2}+3 \cdot 7^{5} h_{7}+7^{6}
$$

and

$$
\mathcal{P}_{7,0} \mathcal{P}_{7,2} \mathcal{P}_{7,6}=22 h_{7}^{4}+2 \cdot 7^{3} h_{7}^{3}+25 \cdot 7^{3} h_{7}^{2}+3 \cdot 7^{5} h_{7}+7^{6}
$$

where

$$
h_{7}:=h_{7}(q)=\frac{1}{q} \prod_{n=1}^{\infty}\left(\frac{\left(1-q^{n}\right)}{\left(1-q^{7 n}\right)}\right)^{4} .
$$

The identities (1.8) and (1.9) suggest that their left hand sides are the "norms" of certain modular functions. If this were the case, then there should be identities associated with the "trace" of such functions. A natural guess would be certain expressions of $\mathcal{P}_{7,1}+\mathcal{P}_{7,3}+\mathcal{P}_{7,4}$ and $\mathcal{P}_{7,0}+\mathcal{P}_{7,2}+\mathcal{P}_{7,6}$ in terms of $h_{7}$. This initial guess turns out to be incorrect. The right functions to consider are functions of the type $\mathcal{P}_{l, i} W_{l, j}$ for some integers $i$ and $j$. For example, we have

$$
\mathcal{P}_{7,0} W_{7,3}+\mathcal{P}_{7,2} W_{7,2}+\mathcal{P}_{7,6} W_{7,1}=-h_{7}^{2}-7 h_{7}-49 .
$$

Note that if we interpret (1.10) as the "trace" of $P_{7,0} W_{7,3}$ then indeed (1.9) is the "norm" since

$$
W_{7,1} W_{7,2} W_{7,3}=1 .
$$

The aim of this article is to establish and prove identities such as (1.10), as well as identities considered in Kolberg's paper.

\section{Properties of the functions $W_{l, j}(z)$}

The functions $W_{l, j}(z)$ were studied by Fine [7] and they occur in the following result of A.O.L. Atkin and P. Swinnerton-Dyer [3, Lemma 6]:

Theorem 2.1. Let $l=6 \lambda \pm 1$ (not necessary prime). Then

$$
(-1)^{\lambda} \frac{\eta(z / l)}{\eta(l z)}=1+\sum_{j=1}^{(l-1) / 2} W_{l, j}(z),
$$

where

$$
\eta(\tau)=q^{1 / 24} \prod_{n=1}^{\infty}\left(1-q^{n}\right), q=e^{2 \pi i z} .
$$

The properties of $W_{l, j}$ are best summarized as follows:

Theorem 2.2. [7], [8, Proposition 2.4.6] Let $l>3$ be a prime and let $k \in \mathbf{Z}$ with $k \not \equiv 0(\bmod l)$. Then

(i) $W_{l, k}=W_{l,-k}=W_{l, k+l}$,

(ii) $W_{l, k}\left(\left(\begin{array}{ll}a & b \\ c & d\end{array}\right) z\right)=e^{12 \pi i k^{2} a b / l} W_{l, a k}$, for $\left(\begin{array}{ll}a & b \\ c & d\end{array}\right) \in \Gamma_{0}(l)$,

(iii) $W_{l, k}(-1 /(l z))=2 \cos (2 k \pi / l)+O(q), q=e^{2 \pi i z}$. 
Fine called the function

$$
F(z)=\Phi\left(W_{l, 1}(z), \cdots, W_{l, \frac{l-1}{2}}(z)\right)
$$

cyclic if $\Phi$ is invariant under $k \mapsto \alpha k$ for every $\alpha$ prime to $l$. If $\Phi$ is a polynomial, he called $F(l, m)$-isobaric whenever each term has "weight" $\equiv m(\bmod l)$, provided that $W_{l, k}$ is assigned "weight" $k^{2}$. From Theorem 2.2 (ii), he concluded that every cyclic function is invariant under $\Gamma_{0}^{0}(l)$, where

$$
\Gamma_{0}^{0}(l)=\left\{\left(\begin{array}{ll}
a & b \\
c & d
\end{array}\right) \mid b \equiv c \equiv 0 \quad(\bmod l)\right\}
$$

and every cyclic, $(l, 0)$-isobaric polynomial is invariant under $\Gamma_{0}(l)$. A simple example of an isobaric $(l, 0)$-polynomial is

$$
\prod_{j=1}^{\frac{l-1}{2}} W_{l, j}
$$

Instead of studying (cyclic) combinations of $W_{l, j}$ as functions on $\Gamma_{0}^{0}(l)$, we consider certain combinations of $W_{l, j}$ as functions on $\Gamma_{1}(l)$. This is motivated by the work of R.J. Evans [6].

If $\alpha_{k} \in \mathbf{Z}$, then from Theorem 2.2,

$$
W_{l, k}^{\alpha_{k}}\left(\left(\begin{array}{ll}
a & b \\
c & d
\end{array}\right) z\right)=e^{12 \pi i\left(k^{2}\right) \alpha_{k} b / l} W_{l, k}^{\alpha_{k}}(z),\left(\begin{array}{ll}
a & b \\
c & d
\end{array}\right) \in \Gamma_{1}(l)
$$

Hence, the following lemma is immediate.

Lemma 2.3. If

$$
\sum_{k=1}^{\frac{l-1}{2}} k^{2} \alpha_{k} \equiv 0 \quad(\bmod l)
$$

then

$$
\prod_{k=1}^{\frac{l-1}{2}} W_{l, k}^{\alpha_{k}}(z)
$$

is a modular function on $\Gamma_{1}(l)$.

Condition (2.1) is precisely the one given in [4, p. 339, (0.56)]. It is known that $\left[12\right.$, p. 106, Theorem 4.2.5 (3)] the index $\left[\Gamma_{0}(l): \Gamma_{1}(l)\right]=l-1$. We choose the coset representatives for $\Gamma_{1}(l)$ in $\Gamma_{0}(l)$ as

$$
\gamma^{k}, 1 \leq k \leq l-1
$$

where

$$
\gamma:=\left(\begin{array}{ll}
g & b \\
c & d
\end{array}\right) \in \Gamma_{0}(l),
$$

with $g$ a primitive root modulo $l$. 
By Theorem 2.2 (ii),

$$
\prod_{k=1}^{\frac{l-1}{2}} W_{l, k}^{\alpha_{k}}(\gamma z)=\prod_{k=1}^{\frac{l-1}{2}} W_{l, g k}^{\alpha_{k}}(z) .
$$

The following theorem then follows.

Theorem 2.4. If $\alpha_{k}$ satisfies (2.1), then

$$
\sum_{j=1}^{\frac{l-1}{2}} \prod_{k=1}^{\frac{l-1}{2}} W_{l, g^{j} k}^{\alpha_{k}}(z)
$$

is a modular function on $\Gamma_{0}(l)$. Moreover, the function has no poles on the upper half plane and at the cusp zero.

Theorem 2.4 is similar to [4, p. 339, Theorem 0.4]. The second statement of Theorem 2.4 follows from Theorem 2.2 (iii).

For convenience, we will refer to functions satisfying the hypothesis of Theorem 2.4 as Fine-Evans functions. We now apply Theorem 2.4 to construct new identities associated with Fine-Evans functions. Recall that when $l=13$, the group $\Gamma_{0}(13)$ has genus 0 and every modular function on $\Gamma_{0}(13)$ can be expressed as a rational function of

$$
h_{13}:=\frac{1}{q} \prod_{n=1}^{\infty}\left(\frac{\left(1-q^{n}\right)}{\left(1-q^{13 n}\right)}\right)^{2} .
$$

In particular, if the only singularity of the function is $i \infty$ then the function is a polynomial of $h_{13}$. It is clear that $\sum_{j=1}^{6} W_{13, j}^{13}(z)$ satisfies the conditions of Theorem 2.4 and hence by the above remark, it is a polynomial in $h_{13}$, namely,

$\sum_{j=1}^{6} W_{13, j}^{13}(z)=h_{13}^{7}+13 h_{13}^{6}+91 h_{13}^{5}+377 h_{13}^{4}+962 h_{13}^{3}+1040 h_{13}^{2}-845 h_{13}-4083$.

We next construct a slightly less obvious example. We know that 2 is a primitive root modulo 13. Suppose in the equation (2.1), we set $\alpha_{1}=\alpha_{5}=1$ and $\alpha_{j}=0$ for $j \neq 1,5$ so that $1^{2}+5^{2} \equiv 0(\bmod 13)$. Then $W_{13,5} W_{13,1}$ is invariant under $\Gamma_{1}(13)$. Now, $W_{13,2.5} W_{13,2}=W_{13,3} W_{13,2}$ and $W_{13,2^{2} \cdot 5} W_{13,2^{2}}=W_{13,6} W_{13,4}$ are the distinct images under the map $W_{13, k} \mapsto W_{13,2^{j} \cdot k}, 1 \leq j \leq 6$. Hence, we conclude that

$$
W_{13,5} W_{13,1}+W_{13,3} W_{13,2}+W_{13,6} W_{13,4}=-1-h_{13} .
$$

This identity is due to Ramanujan [4, p. 373, (8.2)]. Infinite families of identities of the type (2.2) can be constructed using the above method. In particular, Ramanujan's modular equations of degrees 5,7 , and 13 which involve $W_{l, j}$ can be proved in this manner since $\Gamma_{0}(5), \Gamma_{0}(7)$ and $\Gamma_{0}(13)$ has genus 0 .

We now consider $\Gamma_{0}(l)$ with genus $>0$. We want to study the modular functions on $\Gamma_{0}(l)$ with only singularity at $i \infty$. In this case, the functions can no longer be expressed using only $\eta$-quotients. However, we conjecture that these 
functions can be expressed as sums of Fine-Evans functions. This conjecture can also be found in [8, Proposition 2.4.12] and [1, p. 418, third paragraph]. An evidence of this conjecture is (2.2), which can be viewed as expressing $h_{13}$ as a polynomial of Fine-Evans functions.

In what follow, we describe a method of constructing Fine-Evans functions for which every modular function on $\Gamma_{0}(11)$ is a polynomial of these functions. Examples of such functions have already been given in [7], [8] and [3]. Our method here, however, allows us to have a systematic way of constructing such functions with specified order at $i \infty$. We have no proof that this method will always work for a general prime $l$. But given $N$, our search for such functions can be performed in a finite number of steps.

We first observe that the order of the pole at $i \infty$ of $W_{l, j}$ is given by

$$
v_{i \infty}\left(W_{l, j}\right)=\left\{\begin{array}{l}
6 j^{2} / l-j \text { if } 1 \leq j<l / 4 \\
6 j^{2} / l-5 j+l \text { if } l / 4 \leq j \leq \frac{l-1}{2} .
\end{array}\right.
$$

Let $l=11$ and let $C$ be the $5 \times 5$ matrix such that

$$
C_{i, j}=v_{i \infty}\left(W_{11,2^{i-1} j}\right)
$$

since 2 is a primitive root modulo 11 . In order to construct a function

$$
F\left(\alpha_{1}, \alpha_{2}, \alpha_{3}, \alpha_{4}, \alpha_{5}\right)=\sum_{j=1}^{5} \prod_{k=1}^{5} W_{11,2^{j} k}^{\alpha_{k}},
$$

with a specified $v_{i \infty}(F)=-N$, say, we need to know the image of $\mathbf{x}=$ $\left(\alpha_{1}, \cdots, \alpha_{5}\right)$ under $C$ subject to the condition

$$
1^{2} \alpha_{1}+2^{2} \alpha_{2}+3^{2} \alpha_{3}+4^{2} \alpha_{4}+5^{2} \alpha_{5}-11 t=0 .
$$

In other words, we consider the system

$$
\left(\begin{array}{cccccc}
-5 & 2 & 10 & -3 & -4 & 0 \\
2 & -3 & -4 & 10 & -5 & 0 \\
-3 & 10 & -5 & -4 & 2 & 0 \\
10 & -4 & 2 & -5 & -3 & 0 \\
-4 & -5 & -3 & 2 & 10 & 0 \\
1 & 4 & 9 & 16 & 25 & -11
\end{array}\right)\left(\begin{array}{c}
\alpha_{1} \\
\alpha_{2} \\
\alpha_{3} \\
\alpha_{4} \\
\alpha_{5} \\
t
\end{array}\right)=\left(\begin{array}{c}
11 a \\
11 b \\
11 c \\
11 d \\
11 e \\
0
\end{array}\right)
$$

Solving the system, we conclude that

$$
a+b+c+d+e=0
$$


and

$$
\begin{aligned}
& \alpha_{1}=\frac{1}{25} a+\frac{12}{25} b+\frac{13}{25} c+\frac{29}{25} d+\frac{1}{5} k \\
& \alpha_{2}=\frac{16}{25} a+\frac{17}{25} b+\frac{33}{25} c+\frac{14}{25} d+\frac{1}{5} k \\
& \alpha_{3}=\frac{21}{25} a+\frac{2}{25} b-\frac{2}{25} c+\frac{9}{25} d+\frac{1}{5} k \\
& \alpha_{4}=\frac{6}{25} a+\frac{22}{25} b+\frac{3}{25} c-\frac{1}{25} d+\frac{1}{5} k \\
& \alpha_{5}=-\frac{14}{25} a-\frac{18}{25} b-\frac{7}{25} c-\frac{6}{25} d+\frac{1}{5} k
\end{aligned}
$$

where $k \in \mathbf{Z}$. Note that in order to have integral $\alpha_{j}, 1 \leq j \leq 5$, the integers $a, b, c$, and $d$ must satisfy

$$
a+2 b+3 c+4 d \equiv 0 \quad(\bmod 5) .
$$

Our hope is to fix $a, b, c, d, e$ in such a way that only one of them takes the value $-N$ and the rest are $>-N$ and it is clear that this is always possible for $N>1$. By setting $(a, b, c, d, k)=(-2,1,0,0,8),(-3,0,1,0,13),(-4,0,0,1,15)$, we obtain the functions

$$
\begin{aligned}
& F(2,1,0,2,2)=q^{-2}+2 q^{-1}+1+5 q+\cdots, \\
& F(3,2,0,2,4)=q^{-3}+4 q^{-2}+9 q^{-1}+16+22 q+\cdots, \\
& F(4,1,0,2,5)=-q^{-4}-5 q^{-3}-14 q^{-2}-31 q^{-1}-59-86 q-\cdots,
\end{aligned}
$$

respectively.

As an application, we consider the function $\mathcal{P}_{11,6}$. In the next section, we will show that this function is on $\Gamma_{0}(11)$ with $i \infty$ as its only pole. Assuming this for the moment, we conclude, by eliminating the negative $q$-powers in the expansion of $\mathcal{P}_{11,6}$ using the $F^{\prime}$, that

$$
\mathcal{P}_{11,6}=-11 F(4,1,0,2,5)+110 F(3,2,0,2,4)+154 F(2,1,0,2,2)+990 .
$$

In particular, we conclude that

$$
p(11 n+6) \equiv 0 \quad(\bmod 11),
$$

another congruence due to Ramanujan. Similar identities (with different FineEvans functions) for $\mathcal{P}_{11,6}$ are first independently given by Atkin and Hussein [2] and Fine [7]. We have illustrated here that there are infinitely many different expressions for $\mathcal{P}_{11,6}$ and these can be obtained by varying $(a, b, c, d, k)$.

It is interesting to note here that there is no solution for the above problem if exactly one of the numbers $a, b, c, d$ and $e$ is -1 and that the rest is strictly greater than -1 . This turns out to correspond to the fact that there are no functions on $\Gamma_{0}(11)$, with exactly a pole of order 1 at $i \infty$. 


\section{The functions $\mathcal{P}_{l, k}$ and $W_{l, j}$}

The main purpose of this section is to prove (1.10). It will be clear that our method can be used to construct many identities such as (1.10). To prove (1.10), the usual approach is to first establish the behavior of $\mathcal{P}_{7, k}$ and $W_{7, j}$ (this is already given in Theorem 2.2) under $\Gamma_{0}(7)$, verify that the expression on the left hand side of (1.10) is invariant under $\Gamma_{0}(7)$ with pole only at $i \infty$, and conclude that it is a polynomial in $h_{7}$ where

$$
h_{7}=\frac{1}{q} \prod_{n=1}^{\infty}\left(\frac{1-q^{n}}{1-q^{7 n}}\right)^{4} .
$$

However, this method does not tell us how to construct identities such as (1.10). Furthermore, it is not clear how one can obtain the modular behavior of $\mathcal{P}_{7, k}$ under $\Gamma_{0}(7)$.

Proof of (1.10). We check that

$$
W_{l, k}\left(l\left(\begin{array}{cc}
a & b \\
l^{2} c & d
\end{array}\right) z\right)=W_{l, k}\left(\frac{a(l z)+l b}{l c(l z)+d}\right)=W_{l, a k}(l z) .
$$

It follows that for any positive integers $\alpha$,

$$
\sum_{k=1}^{\frac{l-1}{2}} W_{7 l, k}^{\alpha}(l z)
$$

is on $\Gamma_{0}\left(l^{2}\right)$ for any fixed integer $\alpha$. In particular, when $l=7$,

$$
W_{7,1}(7 z)+W_{7,2}(7 z)+W_{7,3}(7 z)
$$

is a modular function on $\Gamma_{0}(49)$ with poles at $i \infty$. Consider the function

$$
G(z):=\frac{\eta(49 z)}{\eta(z)}\left(W_{7,1}(7 z)+W_{7,2}(7 z)+W_{7,3}(7 z)\right) .
$$

This is a function on $\Gamma_{0}(49)$ with pole of order 2 at 0 and a pole of certain order at $i \infty$.

We now introduce the $U(m)$-operator defined on a formal power series $\sum_{n=0}^{\infty} a_{n} q^{n}$ by

$$
\left.\sum_{n=0}^{\infty} a_{n} q^{n}\right|_{U(m)}=\sum_{n=0}^{\infty} a_{m n} q^{n}
$$

It can be checked that

$$
\left.\left(\sum_{n=0}^{\infty} b_{n} q^{m n} \sum_{n=0}^{\infty} a_{n} q^{n}\right)\right|_{U(m)}=\sum_{n=0}^{\infty} b_{n} q^{n} \sum_{n=0}^{\infty} a_{m n} q^{n} .
$$

Since

$$
\prod_{n=1}^{\infty} \frac{1}{\left(1-q^{n}\right)}=\sum_{n=0}^{\infty} p(n) q^{n}
$$


we find that

$$
\begin{aligned}
G(z)=\prod_{n=1}^{\infty}\left(1-q^{49 n}\right)\left(\prod_{n=1}^{\infty}\right. & \frac{\left(1-q^{49 n-21}\right)\left(1-q^{49 n-28}\right)}{\left(1-q^{49 n-14}\right)\left(1-q^{49 n-35}\right)} \sum_{n=0}^{\infty} p(n-1) q^{n} \\
& -\prod_{n=1}^{\infty} \frac{\left(1-q^{49 n-42}\right)\left(1-q^{49 n-7}\right)}{\left(1-q^{49 n-21}\right)\left(1-q^{49 n-28}\right)} \sum_{n=0}^{\infty} p(n-5) q^{n} \\
& \left.-\prod_{n=1}^{\infty} \frac{\left(1-q^{49 n-14}\right)\left(1-q^{49 n-35}\right)}{\left(1-q^{49 n-7}\right)\left(1-q^{49 n-42}\right)} \sum_{n=0}^{\infty} p(n) q^{n}\right) .
\end{aligned}
$$

Using the method illustrated in [10, p. 499-501] together with Theorem 2.2 (iii), we deduce that $\left.G(z)\right|_{U(7)}$ is a modular function on $\Gamma_{0}(7)$ with a pole of order 2 at the cusp 0. Therefore, the function

$$
\left.G(z)\right|_{U(7)} \cdot \frac{\eta^{8}(\tau)}{\eta^{8}(7 \tau)}
$$

is holomorphic everywhere except at $i \infty$ and we conclude that it is a polynomial in $h_{7}$ given by the right hand side of (1.10). (Note that similar argument for $l=11$ allows us to conclude that $\mathcal{P}_{11,6}$ is a function on $\Gamma_{0}(11)$ with just pole at $i \infty$. This is assumed in the previous section). On the other hand, by applying (3.1) to the right hand side of $(3.2)$ and multiplying by $\frac{\eta^{8}(\tau)}{\eta^{8}(7 \tau)}$, we obtain the left hand side of (1.10) and we conclude our proof.

Remarks. We may rewrite the left hand side of (1.10) in terms of Fine-Evans functions, namely,

$$
\mathcal{P}_{7,0} W_{7,3}+\mathcal{P}_{7,2} W_{7,2}+\mathcal{P}_{7,6} W_{7,1}=F_{7}(7,0,0)+7 F_{7}(3,2,0)-27,
$$

where

$$
F\left(\alpha_{1}, \alpha_{2}, \alpha_{3}\right)=\sum_{j=1}^{3} \prod_{k=1}^{3} W_{7,2^{j} k}^{\alpha_{k}} .
$$

In a similar way, we establish the following two identities:

$$
\mathcal{P}_{7,0} W_{7,1}^{2}+\mathcal{P}_{7,2} W_{7,3}^{2}+\mathcal{P}_{7,6} W_{7,2}^{2}=-3 F_{7}(7,0,0)+7 F_{7}(3,2,0)+39,
$$

and

$$
\mathcal{P}_{7,0} W_{7,2}^{4}+\mathcal{P}_{7,2} W_{7,1}^{4}+\mathcal{P}_{7,6} W_{7,3}^{4}=-13 F_{7}(7,0,0)-7 F_{7}(3,2,0)-69 .
$$

This sets up the system of equations

$$
\left(\begin{array}{lll}
W_{7,3} & W_{7,2} & W_{7,1} \\
W_{7,1}^{2} & W_{7,3}^{2} & W_{7,2}^{2} \\
W_{7,2}^{4} & W_{7,1}^{4} & W_{7,3}^{4}
\end{array}\right)\left(\begin{array}{l}
\mathcal{P}_{7,0} \\
\mathcal{P}_{7,2} \\
\mathcal{P}_{7,6}
\end{array}\right)=\left(\begin{array}{ccc}
1 & 7 & -27 \\
-3 & 7 & 39 \\
-13 & -7 & -69
\end{array}\right)\left(\begin{array}{c}
F(7,0,0) \\
F(3,2,0) \\
1
\end{array}\right)
$$


Solving (3.6), we obtain expressions of $\mathcal{P}_{7, k}, k=0,2$ and 6 in terms of $W_{7, j}$, say,

$$
\mathcal{P}_{7, k}=\frac{N_{k}\left(W_{7,1}, W_{7,2}, W_{7,3}\right)}{D_{k}\left(W_{7,1}, W_{7,2}, W_{7,3}\right)},
$$

where $N_{k}(X, Y, Z), D_{k}(X, Y, Z) \in \mathbf{Z}[X, Y, Z]$. These final expressions are then simplified using the Gröbner basis package in Maple V and Ramanujan's identities [4, Chapter 19, (18.8)]

$$
W_{7,1} W_{7,2} W_{7,3}=1 \quad \text { and } \quad W_{7,1} W_{7,2}^{2}+W_{7,1}^{2}+W_{7,3}=0 .
$$

For example, we find that

$$
\mathcal{P}_{7,0} W_{7,3}=W_{7,3}^{7}-2 W_{7,1}^{5} W_{7,3}-10 W_{7,2}^{3} W_{7,3}+3 W_{7,1} W_{7,3}^{3}-14,
$$

an expression similar to (1.5).

\section{Acknowledgement}

This article was completed during the first author's visit to the University of Sussex as a Commonwealth Fellow. He thanks the School of Mathematical Sciences for its hospitality. The authors thank Professors G. E. Andrews and B. C. Berndt for their helpful comments, and Professor F. G. Garvan for bringing to our attention [1] and [8].

\section{References}

[1] A. O. L. Atkin, Explicit gap series at cusps of $\Gamma(p)$, Math. Comp. 22 (1968) 416-419.

[2] A. O. L. Atkin, S. M. Hussain Some properties of partitions. II, Trans. Amer. Math. Soc. 89 (1958), 184-200.

[3] A. O. L. Atkin, P. Swinnerton-Dyer, Some properties of partitions, Proc. London. Math. Soc. (3) 4 (1954), 84-106.

[4] B. C. Berndt, Ramanujan's Notebooks. Part III, Springer-Verlag, New York, 1991.

[5] A. B. Ekin, The rank and the crank in the theory of partitions, D.Phil. Thesis, University of Sussex, 1993.

[6] R. J. Evans, Theta function identities, J. Math. Anal. Appl. 147 (1990), 97-121.

[7] N. J. Fine, On a system of modular functions connected with the Ramanujan identities, Tôhoku Math. J. (2) 8 (1956), 149-164.

[8] F. G. Garvan, Some congruences for partitions that are p-cores, Proc. London Math. Soc. (3) 66 (1993), 449-478.

[9] O. Kolberg, Some identities involving the partition function, Math. Scand. 5 (1957), 77-92.

[10] J. Lehner, Ramanujan identities involving the partition function for the moduli $11^{\alpha}$, Amer. J. Math. 65 (1943), 492-520.

[11] R.P. Lewis, The components of modular forms, J. London Math. Soc. (2) 52 (1995), 245-254.

[12] T. Miyake, Modular forms, Springer-Verlag, Berlin, 1989.

[13] S. Ramanujan, Some properties of $p(n)$, the number of partitions of $n$, Proc. Cambridge Philos. Soc. 19 (1919), 207-210.

[14] H.S. Zuckerman, Identities analogous to Ramanujan's identities involving the partition function, Duke Math. J. 5 (1939), 88-110. 
Department of Mathematics, National University of Singapore, 2 Science Drive 2 , Singapore 117543, Republic of Singapore.

E-mail address: chanhh@math.nus.edu.sg

Department of Mathematics, University of Illinois at Urbana-Champaign, 1409 West Green Street, IL 61801, U.S.A.

E-mail address: hhahn1@math.uiuc.edu

School of Mathematical Sciences, University of Sussex, BN1 9QH, Brighton, U.K.

E-mail address: r.p.lewis@susx.ac.uk

Blk 118, Clementi Street 13, \#14-85, Singapore 120118, Republic of Singapore.

E-mail address: tsl@asean-mail.com 